\title{
Improving Access to Specialized Centers is Not Enough to Mitigate Socioeconomic Disparities in Complex Oncologic Surgery
}

\author{
John Miura, MD, and Giorgos Karakousis, MD \\ Hospital of the University of Pennsylvania, Philadelphia, PA
}

Disparities based on race, socioeconomic status, and insurance status have been demonstrated in cancer care across a multitude of cancer types. ${ }^{1-3}$ These disparities have been manifested in a variety of different ways including differences in access to care and presentation status, postoperative outcomes, and survival. Despite multiple scientific advances in cancer therapy, many disparities persist, reminding us of how medical advances alone cannot serve to equalize care for all patients. Therefore, studies aimed at understanding the source of the disparities are of paramount importance in mitigating inequities in care and improving the lives of all our cancer patients.

In this issue of Annals of Surgical Oncology, Rieser and colleagues ${ }^{4}$ study the impact of socioeconomic differences on the outcomes of patients undergoing cytoreductive surgery (CRS) with heated intraperitoneal chemotherapy (HIPEC) for peritoneal disease of colorectal origin at a high-volume center. Patients with low socioeconomic status (SES) were found more likely to present with synchronous peritoneal disease than those with high SES, suggesting possible barriers to accessing care or delay in diagnosis. Importantly, low-SES patients after CRS-HIPEC had higher rates of immediate postoperative complications and 30-day mortality, a finding that also has been demonstrated for several other cancer surgeries. Notably, although progression-free survival did not differ after CRS-

(C) Society of Surgical Oncology 2021

First Received: 7 January 2021

Accepted: 12 January 2021;

Published Online: 6 February 2021

G. Karakousis, MD

e-mail: Giorgos.Karakousis@pennmedicine.upenn.edu
HIPEC between socioeconomic groups, overall survival was significantly worse in the low-SES group, even after adjustment for other relevant covariates. This suggests that differences in therapeutic interventions or subsequent care rather than tumor biology factors alone may play an important role in the outcome for these patients with advanced-stage disease.

The fact that important disparities were noted between socioeconomic groups even after referral to a high-volume specialized center for CRS-HIPEC highlights the point that access to care is only one piece of the disparity puzzle for complex oncologic surgical cases. Relatively few specialized high-volume CRS-HIPEC centers exist nationally, and only a portion of patients with colorectal peritoneal disease are ultimately referred for consideration of this procedure. Many of these patients are treated with systemic therapy alone for their disease. ${ }^{5-7}$

As the treatment paradigm for colorectal metastasis continues to evolve, the influence that a referring hospital has on the therapeutic options offered cannot be overemphasized. In the Netherlands, where regional therapies are centralized, Rovers et al. ${ }^{6}$ demonstrated that only $13 \%$ of patients with colorectal peritoneal disease diagnosed at non-HIPEC centers ultimately underwent CRS-HIPEC versus $33 \%$ of those with the same diagnosis at a HIPEC center.

The potential barriers to referral identified by the authors were lack of awareness of CRS-HIPEC and a significant number of clinicians not considering CRS-HIPEC as an effective treatment option, both of which are unrelated to socioeconomic status. Increasing awareness within the medical community of the effectiveness and role of CRSHIPEC for peritoneal surface malignancies could perhaps help to mitigate underlying disparities related to socioeconomic factors. Without greater support from clinicians, only 
a small subset of patients will continue to be evaluated for CRS-HIPEC, which undoubtedly would disproportionally affect negatively the number of low-SES patients offered this treatment option.

Additionally, it would be helpful to know (data not presented in the current study) the proportion of patients referred for HIPEC to the institution who actually underwent surgery and whether that selection process had further disparities. With ongoing health care reform and changes in reimbursement structures, the financial pressures on treatment selection will persist.

For appropriately selected patients, the authors demonstrated that repeat CRS-HIPEC was associated with improved OS. Nevertheless, lower-SES patients were less likely to undergo repeat HIPEC. However, this difference was not statistically significant in their multivariate model (odds ratio [OR] $0.45 ; p=0.09$ ).

As a surrogate for SES, insurance status often is reported. In the current study, higher rates of Medicare and Medicaid were reported among the lower-SES cohort. Prior studies have demonstrated that whereas CRS-HIPEC remains profitable among patients with private insurance ( $\$ 14,523$ per patient), net losses of $\$ 17,342$ per patient are noted among Medicare and Medicaid patients, even in the absence of major complications. ${ }^{8}$

As the medical community moves toward a bundled reimbursement structure, hospitals will continue to face financial pressures, especially for costlier therapies. To ensure that financial considerations do not have an undue influence on treatment approaches and that patients regardless of insurance status continue to have access to indicated specialized therapies, reassessment of reimbursement structures is needed.

The current study demonstrated that even after shortterm (30-day) mortality is excluded, important differences persist in long-term mortality between socioeconomic groups. Whether this represents differences in adjuvant treatments (although adjuvant chemotherapy notably was not significantly associated with overall survival in the multivariate analysis), general health maintenance, early re-initiation of therapy upon recurrence or progression, nutritional support, patient's wishes, or other unmeasured factors, is unclear. Nonetheless, these findings underscore the potentially important role that continuity of care by physicians plays in specialized centers for low-SES patients to help maintain continued optimal cancer care well after the complex oncologic surgical procedure is performed. This requires excellent collaboration and communication among clinical care team members including surgeons, oncologists, nutritionists, social workers, home nurses, and family support.

Persistent disparities in cancer surgery and medicine in general are likely multifactorial and no doubt include factors outside the scope of medicine. Nonetheless, the first step toward addressing them is to identify the extent of the problem and wherein these disparities lie. The authors of the current study should be applauded for attempting to delineate various areas of differences in short- and longterm outcomes between socioeconomic groups after CRS and HIPEC. More studies such as this are needed to better understand the root of these disparities across various cancers and procedures and to help us get closer to the goal of delivering high-quality care to all cancer patients.

DISCLOSURE There are no conflicts of interest.

\section{REFERENCES}

1. Reames BN, Birkmeyer NJ, Dimick JB, et al. Socioeconomic disparities in mortality after cancer surgery: failure to rescue. JAMA Surg. 2014;149:475-81.

2. Grubbs SS, Polite BN, Carney J Jr, et al. Eliminating racial disparities in colorectal cancer in the real world: it took a village. J Clin Oncol. 2013;31:1928-30.

3. Polite BN, Adams-Campbell LL, Brawley OW, et al. Charting the future of cancer health disparities research: a position statement from the American Association for Cancer Research, the American Cancer Society, the American Society of Clinical Oncology, and the National Cancer Institute. J Clin Oncol. 2017;35:3075-82.

4. Rieser CJ, Hoehn RS, Zenati M, et al. Impact of socioeconomic status on presentation and outcomes in colorectal peritoneal metastases following cytoreduction and chemoperfusion: persistent inequalities in outcomes at a high-volume center. Ann Surg Oncol. 2021. https://doi.org/10.1245/s10434-021-09627-2.

5. Krell RW, Regenbogen SE, Wong SL. Variation in hospital treatment patterns for metastatic colorectal cancer. Cancer. 2015;121:1755-61.

6. Rovers KP, Simkens GA, Vissers PA, et al. Survival of patients with colorectal peritoneal metastases is affected by treatment disparities among hospitals of diagnosis: a nationwide populationbased study. Eur J Cancer. 2017;75:132-40.

7. Atallah C, Oduyale O, Stem M, et al. Are academic hospitals better at treating metastatic colorectal cancer? Surgery. 2021;169:248-56.

8. Squires MH III, Staley CA, Knechtle W, et al. Association between hospital finances, payer mix, and complications after hyperthermic intraperitoneal chemotherapy: deficiencies in the current healthcare reimbursement system and future implications. Ann Surg Oncol. 2015;22:1739-45.

Publisher's Note Springer Nature remains neutral with regard to jurisdictional claims in published maps and institutional affiliations. 